\title{
Nurses versus physicians' knowledge, attitude, and performance on care for the family members of dying patients
}

\author{
Abdolghani Abdollahimohammad', Mohammadreza Firouzkouhi', Fatemeh Amrollahimishvan ${ }^{2}$ and \\ Nasrollah Alimohammadi ${ }^{3}$ \\ ${ }^{1}$ Nursing and Midwifery School, Zabol University of Medical Sciences, Zabol, ${ }^{2}$ Faculty of Nursing and Midwifery, \\ Guilan University of Medical Sciences, Guilan, and ${ }^{3}$ School of Nursing and Midwifery, Isfahan University of \\ Medical Sciences, Isfahan, Iran
}

Purpose: Nurses and physicians must be competent enough to provide care for the clients. As a lack of knowledge and a poor attitude result in a low performance of delivering care, this study aimed to explore the nurses versus physicians' knowledge, attitude, and performance on care for the family members of dying patients (FMDPs).

Methods: This descriptive study was conducted at the educational hospitals in Isfahan, Iran. The samples were 110 nurses and 110 physicians. The data were collected through a convenience sampling method and using a valid and reliable questionnaire. Results: The average knowledge, attitude, and performance on care for the FMDPs were not significantly different between nurses and physicians ( $p>0.05)$. The majority of nurses (55.4\%) and physician (63.6\%) were at a moderate and a fair level of knowledge of care for the FMDPs. Most of the nurses (81\%) and physicians (87.3\%) had a positive attitude towards caring the FMDPs. Most of the nurses (70\%) and physicians $(86.3 \%)$ had a moderate and fair performance.

Conclusion: Having enough knowledge and skills, and a positive attitude are necessary for caring the FMDPs. Nurses' and physicians competencies must be improved through continuing educational programs and holding international and national conferences with a focus on the palliative care.

Key Words: Family, Nurses, Palliative care, Terminally ill patients

\section{Introduction}

Nurses and physicians compared to other health care providers confront more with stressful and tragic situations such as death and dying [1]. They play a crucial role for the family members of dying patients (DPs) [2] to deliver palliative care [3]. The families and patients are inseparable part of palliative care, which is "an approach that improves the quality of life of patients and their families facing the problem associated with life-threatening illness, through the prevention and relief of suffering by means of early identification and impeccable assessment and treatment of pain and other problems, physical, psychosocial and spiritual" [4].

Families are an important part of the palliative or end-of-life (EOL) care. They bear multiple stresses, which may influence on the dying patients' responses
Received: November 11, 2015 • Revised: December 1, 2015 • Accepted: December 1, 2015 Corresponding Author: Nasrollah Alimohammadi (http://orcid.org/0000-0003-1929-4101) School of Nursing and Midwifery, Isfahan University of Medical Sciences, Isfahan 73461-81746, Iran

Tel: +98.3137927516 Fax: +98.3136699398 email: alimohammadi@nm.mui.ac.ir
Korean J Med Educ 2016 Mar; 28(1): 79-85.

http://dx.doi.org/10.3946/kjme.2016.12

eISSN: 2005-7288

(C) The Korean Society of Medical Education. All rights reserved. This is an open-access article distributed under the terms of the Creative Commons Attribution Non-Commercial License (http:// creativecommons.org/licenses/by-nc/3.0/), which permits unrestricted non-commercial use, distribution, and reproduction in any medium, provided the original work is properly cited. 
and recovery [5]. The needs of patients and their families are intertwined. When a family member encounters a critical situation or life-threatening condition, the whole family system experience disequilibrium [5,6]. In this situation, the healthcare providers, nurses and physicians, have to provide comfort and psychological support for both the families and the patients [1]. Nurses and physicians must also understand the families' needs with regard to hospitalization of their dying patients to help them adapt to this event [5].

The family members assist healthcare providers to assess, diagnose, and treat the patients properly through contributing vital information and supporting the health needs of the patients [7]. Family members also support nurses to diminish the patients' distresses by adapting information before conveying to them [8]. The contributions of the families to care their patients is a need $[1,6,7]$, as well as a valuable interaction among the DPs, families, and nurses [1].

The transition from cure to palliative care is the most challenging stage. Nurses can influence on families' memories when fulfill their needs at this stage [9]. Promoting families' presence [10], proximity and privacy, especially when a loved one is dying or death occurs [9] resulting in reducing anxiety and increasing satisfaction and health outcomes [5].

There is still a paucity of research studies in EOL care for the DPs' families. Nurses and physicians must be competent enough to provide care for the clients. As a lack of knowledge and a poor attitude result in a low performance in delivering care, this study aimed to explore the nurses' and physicians' knowledge, attitude, and performance about care of the families of DPs.

\section{Subjects and methods}

This descriptive study aimed to explore and compare the nurses' and physicians' knowledge, attitude, and performance of care for the family members of dying patients (FMDPs). The study was conducted at educational hospitals, including Al-Zahra, Amin, Shahid Beheshti, Kashani, Noor, Shahid Chamran, Imam Kazem, Sayed-al-shohada, Faiz and Ali Asghar in Isfahan, Iran.

The study populations were the nurses who worked in the medical-surgical and pediatrics wards, emergency departments, and critical care units and the physicians who were specialist in a medical field such as internal medicine. The participants who had lost one of their closed relatives during the last year before the study or had no experience to look after the FMDPs were excluded from the study. However, the volunteer participants were included in the study anonymously. Overall 110 nurses and 110 physicians participated in the study.

The data were collected through a convenience sampling method and using a valid and reliable questionnaire, which was included 24 items to measure knowledge (eight items), attitude (eight items), and performance (eight items) of nurses in caring of the FMDPs (Table 1). The knowledge of nurses was measured using items with three alternative answers: "correct," "wrong," and "do not know." The attitude items were in a 5-point Likert-type scale ranging from absolutely disagree (1) to absolutely agree (5). The alternatives of performance items were "yes," "no," and "did not encounter."

A panel of experts approved the content- and face validity of the questionnaire. A pilot study with 20 participants was conducted to find out any ambiguity in the questionnaire. The reliability of knowledge and 
Table 1. Frequency of Competency of Representing Images

Item

We should honestly answer the questions of the family member about their patients.

We should allow the family members to care their patients in the ward.

The families of dying patients have right to determine the frequency and visit times.

Dying patients will be depressed if their children visit them.

It is a necessity to talk with the families about their grieving.

The family members need emotional preparedness to accept the death of their loved ones.

A family member should be present in the hospital during the last days of their loved ones.

The families should present and touch the corpse of their loved ones.

Table 2. Comparison of Nurses' and Physicians' Knowledge, Attitude, and Performance of Caring for the Families of Dying Patients

\begin{tabular}{llcccc}
\hline \multicolumn{1}{c}{ Profession care } & Nurse $(\mathrm{n}=110)$ & Physician $(\mathrm{n}=110)$ & $95 \% \mathrm{Cl}$ & Independent t-test (df) & $\mathrm{p}$-value \\
\hline Knowledge & $61.06 \pm 20.84$ & $58.48 \pm 16.24$ & -2.36 to 7.52 & $1.02(218)$ & 0.30 \\
Attitude & $75.04 \pm 8.35$ & $74.41 \pm 7.30$ & -1.44 to 2.70 & $0.60(218)$ & 0.55 \\
Performance & $69.15 \pm 27.13$ & $71.55 \pm 21.49$ & -8.87 to 4.06 & $0.73(218)$ & 0.47 \\
\hline
\end{tabular}

Data are presented as mean \pm standard deviation.

$\mathrm{Cl}$ : Confidence interval.

performance items was measured using test-retest method in which the coefficient correlations, which were 0.79 and 0.83 for them, respectively. The reliability of attitude items was tested using Cronbach $\alpha$, which was 0.72 . The data were collected during 3 weeks.

The ethical approval was obtained from the research committee of higher education board of Isfahan Nursing School, Iran (T298-79127). Oral and written consent were taken from the participants before distribution the questionnaires. All collected data were anonymous.

In order to analyze the knowledge data, we assigned a 1 scores for correct answers and a 0 scores for wrong or do not know answers. We also offered a 1 and a 0 score for the correct and wrong performances, respectively. If the participants selected the "did not encounter" to an item, that item was excluded from the total score summation. The summed scores of the knowledge and performance were transformed into a range of 0 and 100 and then scores categorized into three levels: poor ( $0^{-}$ 49), moderate (50-75), and fair (76-100). The attitude's scores were also categorized into three levels: negative (0-33), neutral (34-66), and positive (67-100). The descriptive analyses, including frequencies, mean (standard deviation [SD]), and confidence interval and the inferential statistics, including independent $t$-test was calculated using SPSS version 13.0 (SPSS Inc., Chicago, USA).

\section{Results}

Of the 110 nurses who participated in the study, 65 (59.1\%) were female, and 66 (60\%) got married. Of the physicians, 58 (52.7\%) were male and $82(74.5 \%)$ were married. The average age of nurses was 33.21 (SD, 6.1) years, with an average of $9.41(\mathrm{SD}, 6.45)$ years job experience. The average age and job experience of physician were $34.14(\mathrm{SD}, 6.43)$ and $4.83(\mathrm{SD}, 4.57)$ years, respectively.

Table 2 compares the knowledge, attitude, and per- 
formances of care for the FMDPs between nurses and physicians. There was no significant between nurses and physicians with regard to knowledge, attitude, and performances of care for the FMDPs ( $>>0.05)$. The majority of nurses (55.4\%) and physicians (63.6\%) were at a moderate and a fair level of knowledge in the care for the FMDPs. However, $44.6 \%$ of the nurses and $36.4 \%$ of physicians had a poor knowledge of the families' care (Fig. 1). The participants understood the advantages of the presence of family members in the hospital ward and giving a regular visiting time for both the dying patients and their families. They also knew that death acceptance is easier when family members touch the corpse of their loved one. However, only a quarter of nurses and $11.8 \%$ knew that the importance of discussing about death and dying with the dying patients and their families.

Fig. 1. The Knowledge of the Nurses and Physicians on Caring for the Family Member of Dying Patients

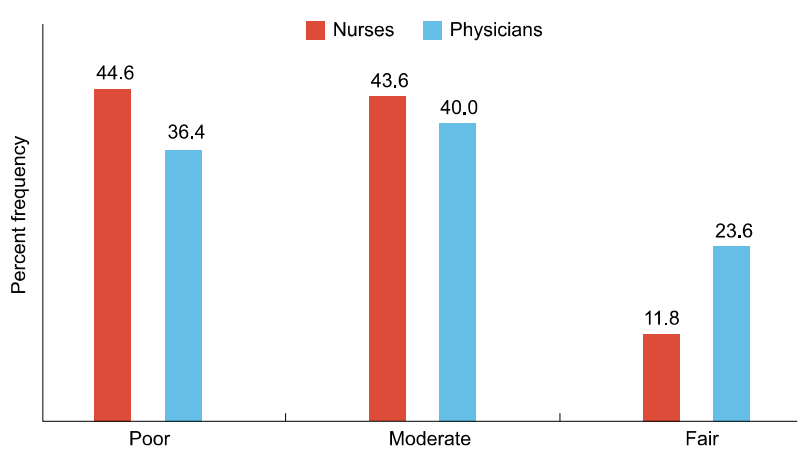

Fig. 2. The Attitude of the Nurses and Physicians towards Caring for the Family Member of Dying Patients

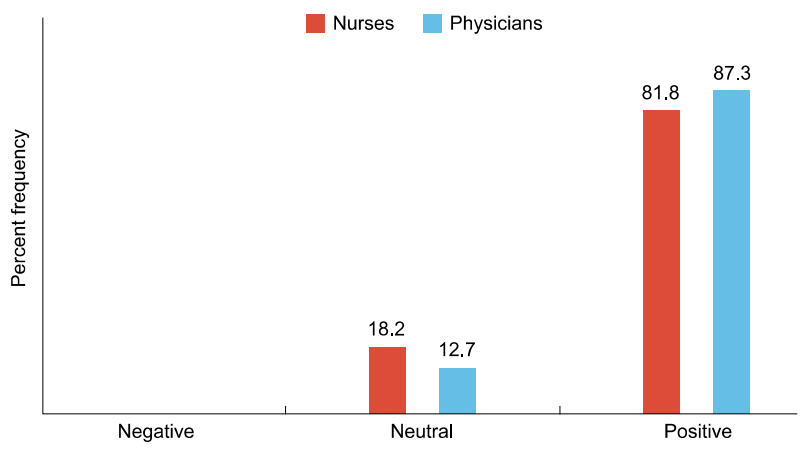

Interestingly, both the nurses (43.6\%) and physicians (43.6\%) realized that if the families could not control their emotions, e.g., anger, they must leave the hospital. Results also showed that the average score of the attitude of both nurses and physicians was at a fair level (Table 2). Most of the nurses (81.8\%) and physicians (87.3\%) had a positive attitude towards the care for the FMDPs. In addition, none of them had a negative attitude regarding care for the families (Fig. 2). Only $43.6 \%$ of nurses and $20.9 \%$ of physicians strongly agreed to the presence of the family members in hospital. However, $16.3 \%$ of nurses believed that the families have the right to visit the patients whenever they aspire. In addition, approximately one-third of nurses and one-fifth of physicians believed that the dying patients would be depressed if the children visit them in the hospital.

The majority the nurses $(63.7 \%)$ and most of the physicians (80.9\%) had a moderate performance for the FMDPs (Fig. 3). Most of them supported the presence of families with their patients and honestly answered to the families' questions regarding their patient status. However, about half of nurses and most of the physicians gave hope to the families, even though they were uncertain about the outcomes. They also encouraged the families to express their feelings about the current situation.

\footnotetext{
Fig. 3. The Performance of the Nurses and Physicians in Caring for the Family Member of Dying Patients
}

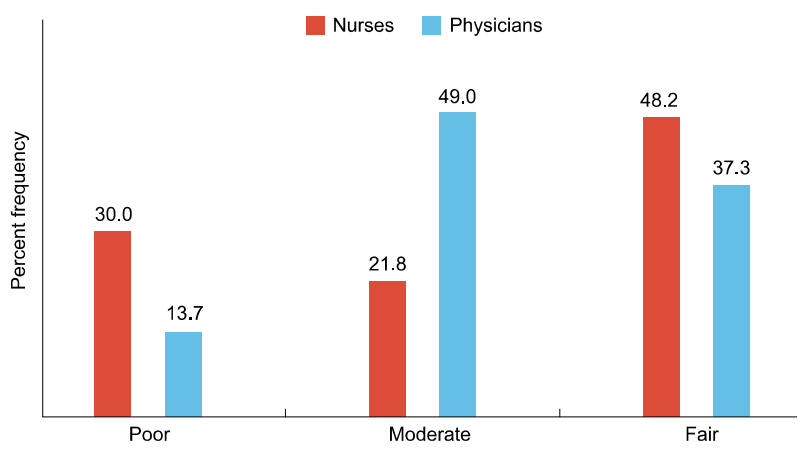




\section{Discussion}

The current study explores and compares the knowledge, attitude, and performance of care for the FMDPs between nurses and physicians. The results showed that the average scores of the nurses and physicians with regard to the care for the FMDPs were not statistically significant and the frequencies of answers to the items were relatively similar.

The majority of the participants had a moderate knowledge of caring for the FMDPs. However, approximately half of them had a poor knowledge about families' care. Besides, most of them were positively eager to care the FMDPs. The nurses were aware that the presence of families in the wards is necessary to fulfill their patients' needs, which result in reducing distress, anxiety, and worries and increasing comfort in both the dying patients and their families. Although there is not a formal duty for the nurses and physicians to care or support the FMDPs in hospitals, reconnecting the families with their patients is an aspect of humanity in a technological environment [10]. The healthcare providers should understand the interdependent needs of the family members and their patients. In the current study, the nurses and physicians supported the families to visit and cooperate in caring for their loved ones. However, the majority of them disagreed with a free visit time as well as permitting the children to visit the patients in hospitals. Such attitudes may associate with the hospitals' rules and regulations, risks, and nursing burdens. Children have right to visit their ill family members in hospitals and the decision whether or not to visit should be left to them [11].

Most of the participants neglected the importance of talking about death and dying with the terminally ill patients and their families. It perhaps indicates a taboo or a lack of communication skills with regard to death and dying issues in the study population. Since a good communication with family members is one of the most important predictor of the quality in EOL care [9], nurses should communicate about dying process in advance and appropriately with the clients to prepare them for critical situations [1]. However, the participants encouraged the families to express their feelings about their current problems and they sincerely responded their questions.

The finding also showed that approximately half of the nurses and three-thirds of physicians gave hope to the families about the patients' outcome. This may indicate a tendency to support families with an uncertainty or indirect communication approach. There are different approaches to discuss EOL issues with the FMDPs. Someone may discuss openly and others may use an indirect approach to give technical or factual information [12]. Cypress [5] indicates that the clients adapt to critical illness through uncertainty, which is the inability to predict outcomes. Uncertainty arises when the clients do not receive enough clues to determine or predict events. However, the family members may exhaust, their interpersonal relationships may deteriorate, and their social support may decrease when they realize the patient's death approaches [13].

The participants realized they cannot tolerate the negative emotions, i.e., anger of the FMDPs and they consequently requested the angry families to leave the wards. Negative emotions are associated with psychological reactions to loss and clients need support of the nurses and physicians in these situations. They should accept it without any negative judgment. Kübler-Ross \& Kessler [14] state that, anger is a useful emotional reaction and should not be suppressed because it facilitates the grieving process, if expresses. Inappropriate reaction or decision may indicate an immature social relation- 
ships and an incompetency in caring for the FMDPs. Nurses and physicians should understand that the transition from cure to palliative care is one of the most challenging stages of life. They can influence on families' memories with fulfilling their needs at this stage [6].

The majority of the participants knew that the acceptance of death would be easier for the family members if they offer a time to touch their loved one's corpse. As grieving process initiates before death and continues after it, the nurses and physicians should provide information for the families according to their sensitivity and level of understanding. Nurses should keep the body in the ward and permit the families to spend time with the body until they are prepared. They should also provide postmortem care based on the clients' rituals [15].

Although the generalization of findings is limited due to a convenience sampling, this study provides valuable information about shortages in knowledge for caring the FMDPs in the nurses and physicians. The results of the participants' performance are also limited due to using a self-report questionnaire. Applying objective methods such as direct observation, Behaviorally Anchored Rating Scale, 360-degree evaluation, and management by objectives may offer data that are more accurate. However, we applied a large multicenter-based sample to reduce the selection bias.

Nurses and physicians must be equipped with professional knowledge and skills concerning the family members' needs on the EOL stage. Nurses' competencies may improve through continuing educational programs and holding palliative care conferences to support the FMDPs. Hospital authorities should also modify the regulations and policies for the dying patients and their families by altering the limitation rule for child visitation, providing a flexible visiting time, and offering a suitable place for the families to increase their presence and cooperation when necessary.

Acknowledgements: We appreciate all the nurses, hospital managers, and university authorities who cooperated and supported the current project.

Funding: None.

Conflicts of interest: None.

\section{References}

1. Zheng RS, Guo QH, Dong FQ, Owens RG. Chinese oncology nurses' experience on caring for dying patients who are on their final days: a qualitative study. Int J Nurs Stud 2015; 52: 288-296.

2. Coombs M. The mourning after: the potential for critical care nurses to improve family outcome and experience in end of life care. Aust Crit Care 2014; 27: 155-156.

3. Montoya-Juarez R, Garcia-Caro MP, Campos-Calderon C, Schmidt-RioValle J, Gomez-Chica A, Marti-García C, Cruz-Quintana F. Psychological responses of terminally ill patients who are experiencing suffering: a qualitative study. Int J Nurs Stud 2013; 50: 53-62.

4. World Health Organization. WHO definition of palliative care [Internet]. World Health Organization; 2011 [cited 2015 March 15]. Available from: http://www.who.int/cancer/palliative/definition/en/.

5. Cypress BS. The lived ICU experience of nurses, patients and family members: a phenomenological study with Merleau-Pontian perspective. Intensive Crit Care Nurs 2011; 27: 273-280.

6. Jack BA, O'Brien MR, Scrutton J, Baldry CR, Groves KE. Supporting family carers providing end-of-life home care: a qualitative study on the impact of a hospice at home service. J Clin Nurs 2015; 24: 131-140.

7. Fry M, Gallagher R, Chenoweth L, Stein-Parbury J. 
Nurses' experiences and expectations of family and carers

of older patients in the emergency department. Int Emerg Nurs 2014; 22: 31-36.

8. Arbour RB, Wiegand DL. Self-described nursing roles experienced during care of dying patients and their families: a phenomenological study. Intensive Crit Care Nurs 2014; 30: 211-218.

9. Fridh I. Caring for the dying patient in the ICU: the past, the present and the future. Intensive Crit Care Nurs 2014; 30: 306-311.

10. Fridh I, Forsberg A, Bergbom I. Doing one's utmost: nurses' descriptions of caring for dying patients in an intensive care environment. Intensive Crit Care Nurs 2009; 25: 233-241.

11. Doka KJ. Children mourning, mourning children. Bristol,
UK: Taylor \& Francis; 1995.

12. Adams JA, Anderson RA, Docherty SL, Tulsky JA, Steinhauser KE, Bailey DE Jr. Nursing strategies to support family members of ICU patients at high risk of dying. Heart Lung 2014; 43: 406-415.

13. Tang ST, Liu LN, Lin KC, Chung JH, Hsieh CH, Chou WC, Su PJ. Trajectories of the multidimensional dying experience for terminally ill cancer patients. J Pain Symptom Manage 2014; 48: 863-874.

14. Kübler-Ross E, Kessler D. On grief and grieving: finding the meaning of grief through the five stages of loss. New York, USA: Scribner; 2007.

15. Williams LS, Hopper PD. Understanding medical surgical nursing. 3rd ed. Philadelphia, USA: F.A. Davis; 2010. 